\title{
Rapid determination of hyaluronic acid concentration in fermentation broth with near-infrared spectroscopy
}

\author{
Qin Dong*, Hengchang Zang**, Lixuan Zang*, Aihua Liu ${ }^{\dagger}$, \\ Yanli Shi ${ }^{\dagger}$ and Hui Zhang* \\ *School of Pharmaceutical Sciences \\ Shandong University and National Glycoengineering Research Center \\ Wenhuaxi Road 44, Jinan 250012, P. R. China \\ ${ }^{\dagger}$ Bloomage Freda Biopharmaceutical Limited Company \\ Tianchen Avenue 678, Jinan 250101, P. R. China \\ \$zanghcw@126.com
}

Received 18 July 2013

Accepted 10 November 2013

Published 24 December 2013

\begin{abstract}
Hyaluronic acid (HA) concentration is an important parameter in fermentation process. Currently, carbazole assay is widely used for HA content determination in routine analysis. However, this method is time-consuming, environment polluting and has the risk of microbial contamination, as well as the results lag behind fermentation process. This paper attempted the feasibility to predict the concentration of HA in fermentation broth by using near infrared (NIR) spectroscopy in transmission mode. In this work, a total of 56 samples of fermentation broth from 7 batches were analyzed, which contained HA in the range of $2.35-9.69 \mathrm{~g} / \mathrm{L}$. Different data preprocessing methods were applied to construct calibration models. The final optimal model was obtained with first derivative using Savitzky-Golay smoothing (9 points window, second-order polynomial) and partial least squares (PLS) regression with leave-one-block-out cross validation. The correlation coefficient and Root Mean Square Error of prediction set is 0.98 and $0.43 \mathrm{~g} / \mathrm{L}$, respectively, which show the possibility of NIR as a rapid method for microanalysis and to be a promising tool for a rapid assay in HA fermentation.
\end{abstract}

Keywords: Near infrared spectroscopy; fermentation; hyaluronic acid.

\section{Introduction}

Hyaluronic acid (HA) is a high-molecular weight biopolysaccharide, discovered in 1934 by Meyer and
Palmer in the vitreous of bovine eyes. It is found in most connective tissues and is particularly concentrated in synovial fluid, the vitreous fluid of the

This is an Open Access article published by World Scientific Publishing Company. It is distributed under the terms of the Creative Commons Attribution 3.0 (CC-BY) License. Further distribution of this work is permitted, provided the original work is properly cited. 
eye, umbilical cords and chicken combs. ${ }^{1} \mathrm{HA}$ is a linear biopolymer with repeating disaccharide units of D-glucuronic acid and N-acetylglucosamine, which are linked alternately by glycosidic linkages. ${ }^{2}$ HA is a valuable material, which has received great interest in the medical, cosmetic and food industries. Traditionally, HA is extracted from rooster combs. In recent years, HA from microbial fermentation is receiving increased attention for avoidance of cross-species viral infection and the use of hazardous solvents. ${ }^{3,4}$

Concentration is one of the most fundamental parameters in fermentation process. Determination of HA concentration in fermentation broth is not only one of the necessary steps when screening HA highyielding bacterial strains, but also a routine measurement during the fermentation process control and optimization. Various methods for determining HA concentration have been previously reported, of which colorimetric methods are often used such as the carbazole assay and the cetyltrimethylammonium bromide (CTBA) turbidimetric method. ${ }^{5,6}$ However, all these methods are extremely laborious and time consuming. Additionally, the operation of the carbazole assay has certain risks since concentrated sulfuric acid is used to treat the samples. Other biochemical methods such as specific hyaluronidase digestion have certain advantages on accuracy and precision, but they are rarely applied to determine large batch fermentation broth samples due to the requirement of experienced personnel to perform and the use of expensive instruments. ${ }^{7}$ Recently, near infrared (NIR) spectroscopy, due to its fast analysis, good precision and accuracy, is increasingly becoming one of the most efficient analytical tools in agricultural, petrochemical and pharmaceutical industries. ${ }^{8-12}$ NIR spectroscopy allows for measurements directly through transparent sample containers, which enables real-time monitoring of large numbers of samples in the production line without affecting the production process, and now becomes more and more considered as an ideal tool for process monitoring, either as part of offline or online quality control.

There are many successful applications in fermentation processes. ${ }^{13-15}$ But so far, no reports were found with regard to the use of NIR spectroscopy to analyze HA directly in a fermentation broth. The aim of the present work was to examine the potential of NIR spectroscopy for such purpose. In comparison with conventional methods, the proposed method was applied easily and could achieve real-time and in situ monitoring, which is suitable for industrialized production.

\section{Materials and Methods}

\subsection{Chemical analysis}

\subsubsection{Preparation of standards}

About $50 \mathrm{mg}$ of D-glucuronic acid of analytical grade, previously dried to constant mass at $105^{\circ} \mathrm{C}$, were dissolved in distilled water of $100 \mathrm{~mL}$ volume. This solution was 10 -fold diluted to obtain the $50 \mu \mathrm{g} / \mathrm{mL}$ reference solution.

To volumes of $0.0,0.2,0.4,0.6,0.8,1.0 \mathrm{~mL}$ of the reference solution in capped test tubes, water was added to a final volume of $1.0 \mathrm{~mL}$, respectively. The test tubes were placed in ice water and cooled to $4{ }^{\circ} \mathrm{C}$. About $5 \mathrm{~mL}$ of freshly prepared sulfuric acid with sodium borate $(4.77 \mathrm{~g}$ disodium tetraborate of analytical grade in $500.0 \mathrm{~mL}$ sulfuric acid of high grade purity) were added to each test tube. The test tubes were closed with caps, shaken and placed in boiling water bath for $10 \mathrm{~min}$, then cooled to room temperature, and to each test tube $0.2 \mathrm{~mL}$ of carbazole solution $(0.125 \mathrm{~g}$ of carbazole in $100.0 \mathrm{~mL}$ of absolute alcohol) was added. The tubes were recapped, shaken and put again in a boiling water bath for $15 \mathrm{~min}$. After this, they were cooled to room temperature and the absorbance of solutions was measured at $530 \mathrm{~nm}$ against a blank sample.

\subsubsection{Sample preparation}

HA production was performed in a fermenter with a working volume of 4000 L. Streptococcus equi subspecies. zooepidemicus was used as the HA producer; the main culture medium include glucose, peptone, yeast extract, $\mathrm{Na}_{2} \mathrm{HPO}_{4}, \mathrm{MgSO}_{4} \cdot 7 \mathrm{H}_{2} \mathrm{O}$ and $\mathrm{NaC} 1$. Fermentation was operated at $37^{\circ} \mathrm{C}$ and $\mathrm{pH} 7.0$ (controlled automatically with $3 \mathrm{M} \mathrm{NaOH}$ ). The aeration rate was $1 \mathrm{vvm}$ and the agitation speed was $400 \mathrm{rpm}$. The fermentation time for each batch was about $24 \mathrm{~h}$, depending on the $\mathrm{pH}$ decreasing rate and content of residual sugar, which was used to judge the fermentation end point. Samples were collected at regular intervals (approximately $2 \mathrm{~h}$ ) after being cultured for $10 \mathrm{~h}$. Finally, 56 samples from 7 batches (8 samples of each batch) were collected.

The collected broth was diluted five-fold with purified water, and then mixed with four-fold 
Rapid determination of $H A$ concentration in fermentation broth

Table 1. HA concentrations in fermentation broth at different times.

\begin{tabular}{lcccccccc}
\hline & \multicolumn{8}{c}{ HA concentration $(\mathrm{g} / \mathrm{L})$} \\
\cline { 2 - 9 } Batch no. & $10 \mathrm{~h}$ & $12 \mathrm{~h}$ & $14 \mathrm{~h}$ & $16 \mathrm{~h}$ & $18 \mathrm{~h}$ & $20 \mathrm{~h}$ & $22 \mathrm{~h}$ & End point \\
\hline 101204 & 3.82 & 5.46 & 6.53 & 7.48 & 7.75 & 8.34 & 8.73 & 8.79 \\
101205 & 2.35 & 4.33 & 6.08 & 7.13 & 8.24 & 8.96 & 9.45 & 9.69 \\
101206 & 3.26 & 5.09 & 6.48 & 7.35 & 8.18 & 8.63 & 8.91 & 8.89 \\
101207 & 2.35 & 4.37 & 6.06 & 6.80 & 7.60 & 8.08 & 9.47 & 9.45 \\
101208 & 2.60 & 4.69 & 6.29 & 7.13 & 8.04 & 8.62 & 8.99 & 8.96 \\
101209 & 2.68 & 4.76 & 6.22 & 7.35 & 8.66 & 9.10 & 9.51 & 8.57 \\
101210 & 2.54 & 4.45 & 6.02 & 7.35 & 8.31 & 8.78 & 9.46 & 9.69 \\
\hline
\end{tabular}

volumes of absolute ethanol. The sediment generated was collected, and then dissolved in purified water. The HA concentration was determined as described above and could be calculated based on the standard calibration curve and the dilution ratio. Table 1 summarizes the reference data for the HA concentrations as measured by chemical analysis.

\subsection{Spectral measurement}

The collected fermentation broth was poured into a glass cuvette of 5-mm optical pathlength and then scanned with a NIRS Antaris II spectrometer (Thermo Fisher Scientific Inc., USA) from 10,000$4000 \mathrm{~cm}^{-1}$, in transmission mode with $8-\mathrm{cm}^{-1}$ resolution. The reference was taken before each scan. To increase the signal-to-noise ratio, both reference and sample spectra were averaged from 64 scans and the gain was auto-optimized. Two spectra of each sample were collected to eliminate the error caused by sample loading.

\subsection{Spectral preprocessing and wavelength selection}

A key first step in modeling is the identification of those wavelength regions where significant spectral change occurs between samples with differing levels of the analyte to be determined. ${ }^{16}$ It is especially important for microanalysis of a complex system.

Many factors affect the spectra through changes in the physico-chemical properties of the culture medium. The increasing biomass not only changes the chemical composition of the matrix but also affects the physical properties like color, density and viscosity of the cultivation medium. Appropriate spectral preprocessing method can significantly remove, reduce or standardize these disturbances and enhance the useful spectral information. In this study, first and second derivative, Savitzky-Golay smoothing with different points, Norris smoothing with different points and several combinations of the above methods were applied to try to find the spectral regions that were correlated to change the HA concentration for improving the accuracy and precision of the calibration model. Due to the strong absorption of water and noise, absorbance larger than 3.5 were removed during spectral processing.

\subsection{Model calibration and validation}

Five batches of 40 samples were selected as calibration set (see Table 2). The partial least squares (PLS) method was used to build calibration models and a leave-one-block-out cross-validation method was used as the model selection criterion. ${ }^{17}$ The optimal number of latent variables for constructing the models was chosen from the minimum of the plot as a function of root mean square error of cross validation (RMSECV) versus the number of latent variables. ${ }^{18}$

In order to corroborate the predictive ability of the established PLS model, the prediction set of the rest of the two batches was used and those HA

Table 2. The reference measurements and sample numbers in calibration set and prediction set.

\begin{tabular}{lccccc}
\hline \multicolumn{1}{c}{ Set } & $n^{\mathrm{a}}$ & $\begin{array}{c}\text { Maximum } \\
\text { value } \\
\left(\mathrm{g} \cdot L^{-1}\right)\end{array}$ & $\begin{array}{c}\text { Minimum } \\
\text { value } \\
\left(\mathrm{g} \cdot L^{-1}\right)\end{array}$ & $\begin{array}{c}\text { Mean } \\
\text { value } \\
\left(\mathrm{g} \cdot L^{-1}\right)\end{array}$ & $\mathrm{SD}{ }^{\mathrm{b}}$ \\
\hline Calibration set & 40 & 9.69 & 2.35 & 6.98 & 2.27 \\
Prediction set & 16 & 9.51 & 2.68 & 7.11 & 1.99 \\
\hline a $n=$ number of samples. & & & \\
${ }^{\mathrm{b}} \mathrm{SD}=$ standard deviation.
\end{tabular}


concentration values obtained by NIR prediction were compared with the results of the chemical analysis. The quality of the models was assessed in terms of the $R_{p}$ and RMSEP value. In addition, the residual predictive deviation (RPD) defined as the ratio of the standard deviation in the validation set to the standard error of validation was used to further evaluate the performance of the calibration model.

\subsection{Software}

The spectral data obtained from the RESULT 3.3.517 software (Thermo Fisher Scientific Inc., USA) were imported to Unscrambler 7.5 (Camo ASA, Oslo, Norway) and subsequently exported to Matlab 7.10 (The MathWorks Inc., Natick, MA, USA). All multivariate data analysis was performed in Matlab.

\section{Results and Discussion}

\subsection{Spectral investigation}

Figure 1 shows the spectra of the samples collected at different times throughout the fermentation. All spectra display quite similar trends, and it is difficult to find differences in the raw spectra due to the high degree of band overlapping.

After applying different spectral preprocessing methods, it was found with a first derivative using Savitzky-Golay smoothing (9 points window, second order polynomial), the absorbance values at $6000-5970 \mathrm{~cm}^{-1}$ that were detected exhibiting obvious HA concentration correlation (see Fig. 2).

\subsection{Calibration models}

The number of samples within the blocks was eight. The number of latent variables finally chosen was

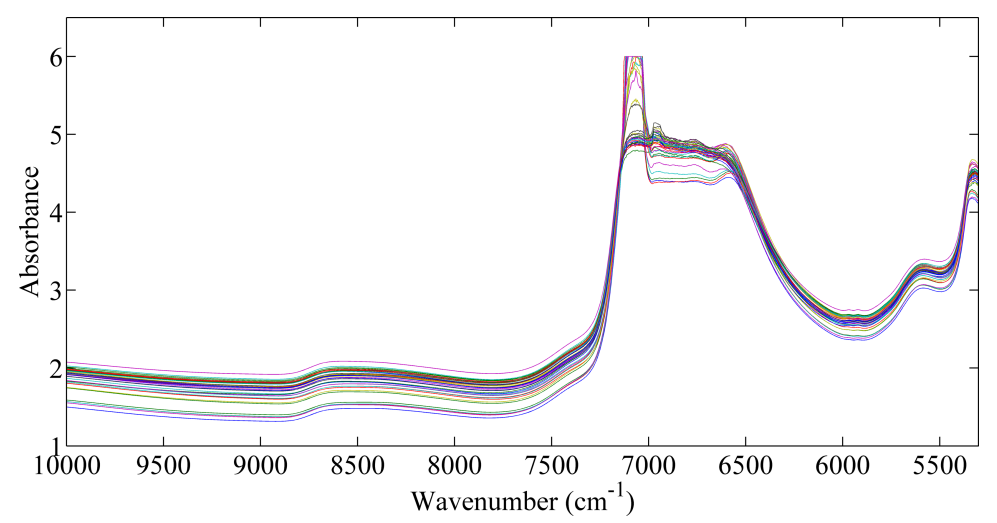

Fig. 1. Original raw NIR spectra of 56 samples taken from HA fermentation broth.

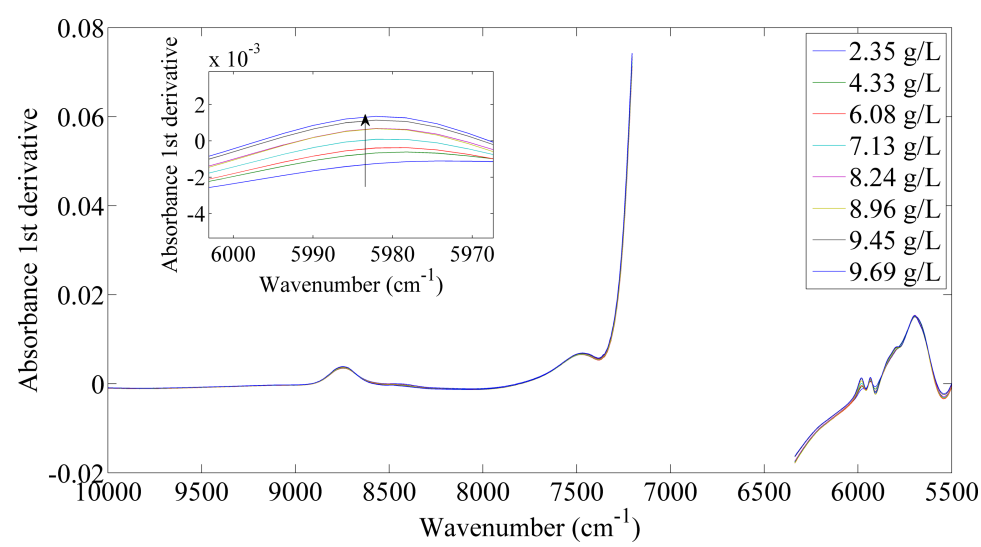

Fig. 2. Plot of first derivative spectra using Savitzky-Golay smoothing (9 points window and second-order polynomial) of HA fermentation broth. In the small inserted graph, the change of absorbance with HA concentration is shown. The upward-pointing arrow indicated increasing HA concentration. 


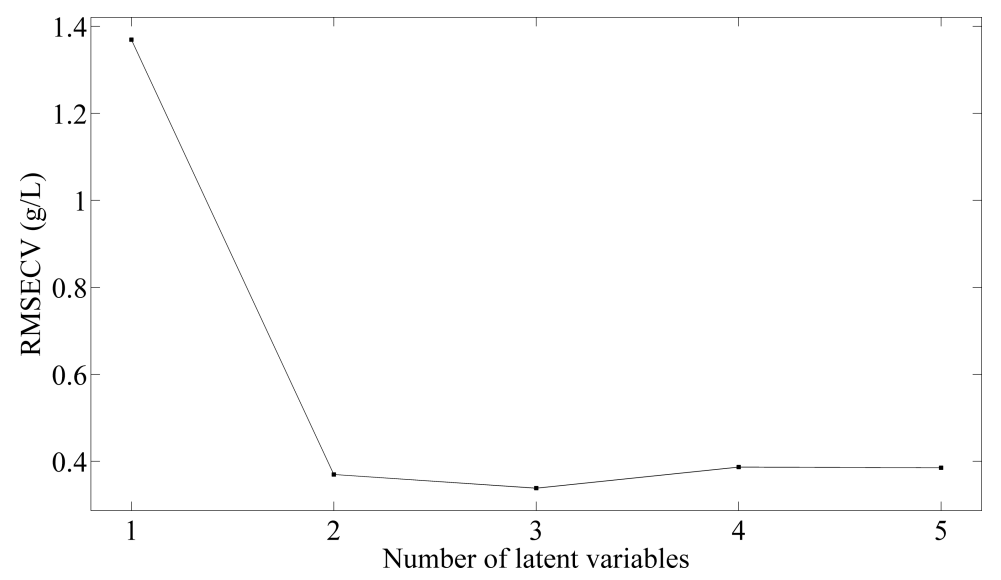

Fig. 3. Effect of the number of latent variables on RMSECV for PLS calibration.

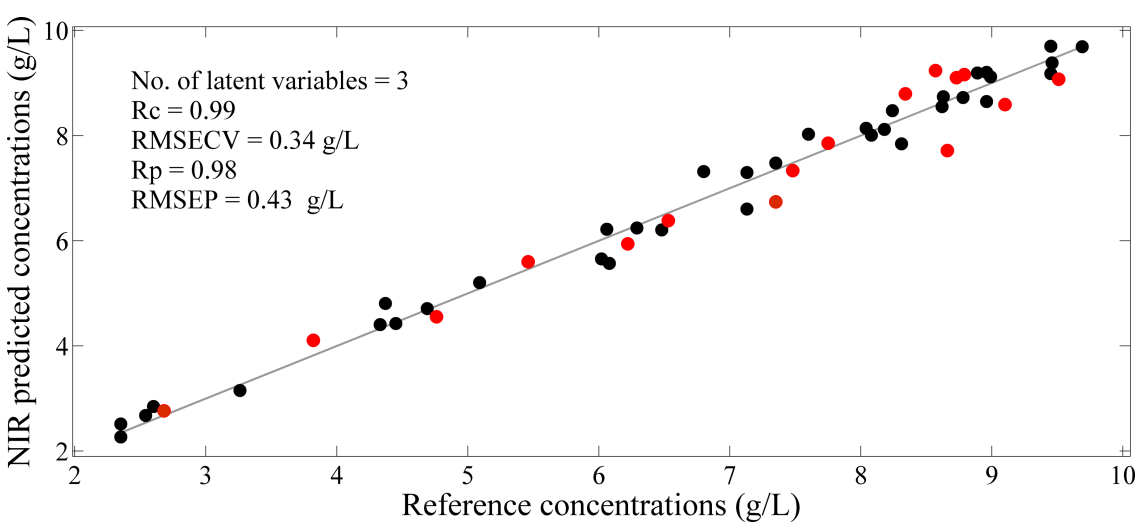

Fig. 4. Reference concentrations measured versus NIR predicted by PLS in the calibration and prediction set, respectively (black circles represent calibration set; red circles represent the prediction set).

three, as shown in Fig. 3. In Fig. 4(a), scatter plot is shown with a high correlation between reference concentrations measured and NIR predicted from the respective calibration and prediction set by the optimum PLS model with a correlation coefficient for the calibration set of $R_{c}=0.99$ and RMSECV of $0.34 \mathrm{~g} / \mathrm{L}$, see in Table 3 .

\subsection{External validation}

The external validation was performed with the samples of prediction set. The $R_{p}, \mathrm{RMSEP}$ and

Table 3. The performance of PLS model within 6000$5970 \mathrm{~cm}^{-1}$ and parameters adopted.

\begin{tabular}{ccccccc}
\hline PLS & & & & & & \\
parameters & $R_{c}$ & RMSECV & $R_{p}$ & RMSEP & PCs & $\mathrm{RPD}^{\mathrm{a}}$ \\
\hline Values & 0.99 & $0.34 \mathrm{~g} / \mathrm{L}$ & 0.98 & 0.43 & 3 & 4.63 \\
\hline
\end{tabular}

${ }^{\mathrm{a}} \mathrm{RPD}=$ the residual predictive deviation.
RPD value were shown in Table 3. The concentration results predicted by calibration model were compared with the results of the chemical analysis with values quite acceptable (see Fig. 4 ). RPD value was greater than 3 which demonstrated the robustness and good performance of the calibration model; since RPD is greater that 3 is considered as good predication. ${ }^{19,20}$

\section{Conclusions}

Although the fermentation matrices were changing constantly and the concentration of HA in the broth was very low, NIR spectroscopy showed an obvious advantage as a rapid nondestructive method for determining HA concentrations in a fermentation broth, based on a narrow spectral region of 6000 $5970 \mathrm{~cm}^{-1}$ to establish the calibration model.

NIR spectroscopy is applicable for microanalysis and has the potential to dramatically reduce the 
analytical time and cost of analysis, especially when the measurement on a large number of batch fermentation broth samples is performed. And the development of such NIR technique is expected to provide attractive opportunities for efficient realtime monitoring and process control during HA fermentation. To establish a practical model in production and truly realize the online monitoring and determination in a very short period of time, further studies need to be performed including improving the calibration specificity, accuracy, robustness, interpreting the spectral changes occurring during fermentation and installing fiberoptics probe on fermentator. By then, it will truly achieve the time-consuming purpose.

\section{Acknowledgments}

This work has been financially supported by the National Glycoengineering Research Center of China, the 863 program (Hi-tech research and development program of China) under contract No. 2012AA021505 and the National Training Programs of Innovation and Entrepreneurship for Undergraduates (No. 201210422079). The authors wish to thank Bloomage Freda Biopharm. Co., Ltd. for the supply of laboratory space and HA samples, and Thermo Fisher Scientific for free use of the FTNIR spectrometer.

\section{References}

1. K. Kakehi, M. Kinoshita, S. Yasueda, "Hyaluronic acid: Separation and biological implications," J. Chromatogr. B Analyt. Technol. Biomed. Life Sci. 797, 347-355 (2003).

2. B. Weissmann, K. Meyer, P. Sampson, A. Linker, "Isolation of oligosaccharides enzymatically produced from hyaluronic acid," J. Biol. Chem. 208, 417-429 (1954).

3. B. F. Chong, L. M. Blank, R. Mclaughlin, L. K. Nielsen, "Microbial hyaluronic acid production," Appl. Microbiol. Biotechnol. 66, 341-351 (2005).

4. L. Jr. Lapcík, L. Lapcík, S. De Smedt, J. Demeester, P. Chabreček, "Hyaluronan: Preparation, structure, properties, and applications," Chem. Rev. 98, 26632684 (1998).

5. T. Bitter, H. M. Muir, "Quantification of hyaluronan in pharmaceutical formulations using high performance capillary electrophoresis and the modified uronic acid carbazole reaction," Anal. Biochem. 4, 330-334 (1962).

6. Y. H. Chen, Q. Wang, "Establishment of CTAB turbidimetric method to determine hyaluronic acid content in fermentation broth," Carbohydr. Polym. 78, 178-181 (2009).

7. Q. S. Gu, K. Yan, Hyaluronan-Based Biomaterials in Clinical Medicine, 1st Edition, The Second Military Medical University Press, Shanghai (2003).

8. P. Baptista, P. Felizardo, J. C. Menezes, M. J. N. Correia, "Multivariate near infrared spectroscopy models for predicting the iodine value, CFPP, kinematic viscosity at 40 degrees $\mathrm{C}$ and density at 15 degrees C of biodiesel," Talanta 77, 144-151 (2008).

9. F. Liu, Y. He, L. Wang, H. Pan, "Feasibility of the use of visible and near infrared spectroscopy to assess soluble solids content and pH of rice wines," J. Food Eng. 83, 430-435 (2007).

10. F. Liu, Y. He, "Classification of brands of instant noodles using Vis/NIR spectroscopy and chemometrics," Food Res. Int. 41, 562-567 (2008).

11. J. Luypaert, D. L. Massart, Y. V. Heyden, "Nearinfrared spectroscopy applications in pharmaceutical analysis," Talanta 72, 865-883 (2007).

12. S. Satya, R. M. Roehner, M. D. Deo, F. V. Hanson, "Estimation of properties of crude oil residual fractions using chemometrics," Energy Fuels 21, 9981005 (2007).

13. A. E. Cervera, N. Petersen, A. E. Lantz, A. Larsen, K. V. Gernaey, "Application of near-infrared spectroscopy for monitoring and control of cell culture and fermentation," Biotechnol. Prog. 25, 1561-1581 (2009).

14. M. Scarff, S. A. Arnold, L. M. Harvey, B. NcNeil, "Near infrared spectroscopy for bioprocess monitoring and control: Current status and future trends," Crit. Rev. Biotechnol. 26, 17-39 (2006).

15. D. Landqrebe, C. Haake, T. Höpfner, S. Beutel, B. Hitzmann, T. Scheper, M. Rhiel, K. F. Reardon, "On-line infrared spectroscopy for bioprocess monitoring," Appl. Microbiol. Biotechnol. 88, 11-22 (2010).

16. I. Giavasis, I. Robertson, B. McNeil, L. M. Harvey, "Simultaneous and rapid monitoring of biomass and biopolymer production by Sphingomonas paucimobilis using Fourier transform-near infrared spectroscopy," Biotechnol. Lett. 25, 975-979 (2003).

17. J. Kohonen, S. P. Reinikainen, K. Aaljoki, A. Perkiö, T. Väänänen, A. Höskuldsson, "Multi-block methods in multivariate process control," J. Chemom. 22, 281-287 (2008).

18. S. Wold, M. Sjöström, L. Eriksson, "PLS-regression: A basic tool of chemometrics," Chemometr. Intell. Lab. Syst. 58, 109-130 (2001). 
19. T. Fearn, "Assessing calibrations: SEP, RPD, RER and R2 RER and R," NIR News 13, 12-14 (2002).

20. D. Cozzolino, M. Kwiatkowski, M. Parker, W. Cynkar, R. Dambergs, M. Gishen, M. Herderich,
"Predication of phenolic compounds in red wine fermentations by visible and near infrared spectroscopy," Anal. Chim. Acta. 513, 73-80 (2004). 